\title{
ISSUES IN STRUCTURAL INVESTIGATIONS: VIBRATION BASED APPROACH VS. ELASTIC WAVE PROPAGATION APPROACH
}

\author{
Sanjay Sengupta ${ }^{1}$, Aloke Kumar Datta ${ }^{2}$, Pijush Topdar ${ }^{3}$ \\ ${ }^{\text {I}}$ Assistant Professor, Dr. B.C Roy Engineering College, Durgapur -713206, India and Part Time Research Scholar, \\ National Institute of Technology, Durgapur, Pin-713209, India (sanswati2004@gmail.com) \\ ${ }^{2}$ Associate Professor, National Institute of Technology, Durgapur, Pin-713209, India (dattarec@yahoo.com) \\ ${ }^{3}$ Assistant Professor, National Institute of Technology, Durgapur, Pin-713209, India)
}

\begin{abstract}
Synopsis
Structural investigations are often related to identification of damages in structures. For survival of the structures through their design lives with full capacity, structural health monitoring (SHM) techniques are used for identification and remedy of damages. SHM is generally carried out in three steps viz periodic visual inspection followed by non destructive testing and sensor based continuous monitoring. The sensor based continuous monitoring, as suggested by the available literature, is based mainly on two different approaches such as vibration based and wave propagation based. One of the potential wave propagation based approaches for SHM is Acoustic Emission (AE) technique through which the location of the damages can be predicted in real time. On the contrary, vibration based approach may be effectively used for damage quantification as it considers the structural properties e.g. stiffness, damping etc. unlike wave propagation based approach. In this study, the commonly used forcing functions for numerical simulation of the AE source, as used in the wave propagation based approach, has been considered to create an internal excitation in the structure causing vibration of the structure. The displacement responses are studied for thin plate using finite element method and compared with the available response data from elastic wave propagation technique. The results are found to follow similar pattern.
\end{abstract}

Keywords: Structural health monitoring, Identification of damages, Acoustic Emission (AE) technique, Finite Element Method, Displacement Response

\section{INTRODUCTION}

In contemporary research, management of costly infrastructure is an area of growing interest. In this context, up keeping of such infrastructural facilities in general and physical infrastructures in particular is extremely important from techno-economic point of view of a nation. Any sort of deviation in their normal functioning is a major concern to the engineering/scientific community. Such deviations/ malfunctioning are often caused by structural damage which arise due to faulty design/construction, overburden load, ageing effect and natural calamity. Structural health monitoring (SHM) techniques are the first step towards investigations and identification of such damages. SHM is generally carried out in three steps viz periodic visual inspection followed by non destructive testing and sensor based continuous monitoring. As suggested by the available literature, there are primarily two approaches for the sensor based continuous monitoring - vibration based and wave propagation based.

One of the potential wave propagation based approaches for SHM is Acoustic Emission (AE) technique. Acoustic emission (AE) is the generation of transient elastic waves due to sudden redistribution of stresses in a material. $\mathrm{AE}$ technique involves the recording of such waves, known as "AE waves", by means of sensors, called as "AE sensors", placed at different locations on the surface of the material and analyzing the recorded data for information about the source. In engineering structures, it is found, that defects like generation of cracks, yielding, failure of bonds, fibre failure etc. generate $\mathrm{AE}$ waves. Therefore, $\mathrm{AE}$ technique, as a non-destructive technique (NDT), has the potential to be utilized for extracting the details, such as location, type and extent of such structural defects as above. In this context, it is worth mentioning that all the other NDTs for structural investigation requires external excitations while the sensors record the relevant data with regard to the response. The $\mathrm{AE}$ technique does not require any external excitation for recording sensor data; instead the energy arising from the generation of the defect within a structure, in real time, is utilized. Thus the internal damages need special procedures like $\mathrm{AE}$ technique for their diagnosis, unlike the surface damages which are visible and can be easily investigated.

Ono and Ohtsu [1] have provided a generalized theory of Acoustic Emission (AE) based on the theory of elastodynamics and dislocation models. Improving upon the generalized theory on AE, Ohtsu and Ono [2] presented the relationship between the $\mathrm{AE}$ source representation and the moment tensor. In the generalized theory of $\mathrm{AE}$ it is found that information about AE sources can be extracted from the waveforms. Gorman and Prosser [3] proposed an approach for interpretation of $\mathrm{AE}$ signals in terms of extensional and flexural mode of plate waves which may be useful in determining $\mathrm{AE}$ source orientation. In recent years researchers have tried to apply the Finite Element Analysis for damage localisation using $\mathrm{AE}$ technique. Gary and 
Hamstad [4] proposed a two dimensional cylindrical symmetric dynamic finite element method (DFEM) for thin plate specimens to model AE source in a more general way than the Pencil-lead-break. The problem has been considered as an initial boundary value problem for elastic wave propagation in an isotropic plate. The pencil-lead break is a valuable source for simulation of $\mathrm{AE}$ source experimentally. However, the limitations for this source are that it can be only applied to outer surface as out-of-plane source and the range of the source rise times and source dimensions are limited. To overcome these limitations, in the proposed model the source force versus time characteristics are varied and the time dependent displacement fields for the far-filed are captured. The AE source is simulated in the DFEM with a normal stress function after Breckenridge et al. [5]. Based on the elastic wave propagation based approach researchers have tried using more refined model for finite element further to the proposed model by Gary and Hamstad [4]. Hamstad et al. [6] used 3-D DFEM to model AE source. Hamstad et al. [7] extended 2-D axi-symmetric DFEM method to consider more complicated AE source as a buried transient dipole. Prosser et al. [8] validated the 3-D DFEM method experimentally for both normal and oblique edge reflections. Prosser et al. [9] used Mindlin Plate Theory (MPT) for predicting flexural plate mode acoustic emission waveforms in thin plates and compared the results with DFEM. Sause and Horn [10] used finite element simulation approach for investigating the acoustic emission waveforms due to cracking in carbon fibre reinforced plastic (CFRP) specimens. Sause and Horn [11] investigated the influence of microscopic elastic properties and the geometry of the AE source by finite element simulation. Sause [12] extended the studies done by Sause and Horn [11] on CFRP to CFRP with internal damage. The prospect and future of $\mathrm{AE}$ technique in structural investigations can be traced in detail in the recent study conducted by Sengupta et al [13]. Study of literature thus indicates that the location of the damage in real time can be obtained using the numerical simulation of the process of propagation of $\mathrm{AE}$ wave as elastic wave. However, in the numerical model of elastic wave propagation, the structural properties like stiffness, damping etc. are not considered. These properties are considered to be very important for structural damage quantification. In the numerical modelling using vibration based approach such properties are considered. Hence, the damage quantification may be more effectively done using the vibration based approach than the wave propagation based approach.

The previous researchers [4] have tried to investigate structural damage using $\mathrm{AE}$ technique modelled as wave propagation problem. Here an effort is made to represent the aforesaid work as structural vibration problem. The structure is investigated using finite element method (FEM) in dynamic condition. The commonly used forcing function for numerical simulation of the AE source, as used in the wave propagation based approach, is used. This is considered to create an internal excitation in the structure. The time dependent displacement response obtained due to the vibration of the structure is compared with the available response data from elastic wave propagation technique.

\section{MATHEMATICAL FORMULATION}

Based on the above, the response of a plate is studied under an excitation similar to an AE signal that simulates a crack in the plate. The governing equation for such analysis is given by:

$$
[M]\{\ddot{u}\}+[C]\{\dot{u}\}+[K]\{u\}=\{F(t)\}
$$

where, $[\mathrm{M}],[\mathrm{C}],[\mathrm{K}],\{\ddot{u}\},\{\dot{u}\},\{u\},\{F(t)\}$ are the mass matrix, damping matrix , stiffness matrix, nodal acceleration vector, nodal velocity vector, nodal displacement vector, nodal load vector respectively. The crack at the centre of the plate is simulated by a forcing function equivalent to an $\mathrm{AE}$ source [5] as follows:

$$
\begin{array}{rlrl}
f(t) & =\frac{t}{T} & & t \leq T \\
& =1 & t>T
\end{array}
$$

In this case damping is neglected. Hence the governing equation is reduced to:

$$
[M]\{\ddot{u}\}+[K]\{u\}=\{F(t)\}
$$

The analysis is done using finite element approach. The governing equation, as above, is solved by time integration scheme following Newmark's $\beta$ method, which is an explicit time integration technique [14]. In the present study, the values of $\alpha$ and $\psi$ are taken as 0.25 and 0.50 respectively. A higher order rectangular element [15] is used. The element is having four nodes at its four corners and each node is having eight degrees of freedom (u, v, w, w, x, w,y, w, xy, $\gamma \mathrm{x}, \gamma \mathrm{y})$ .To implement the above mathematical formulation, a computer code is developed using FORTRAN.

\section{NUMERICAL EXAMPLE}

The mathematical formulation as above is implemented by solving numerical problems. To validate the present formulation against the established results that are available for plate analysis by AE approach [4] a $3.05 \mathrm{~mm}$ thick isotropic plate measuring $1.2 \mathrm{~m} \mathrm{x} 1.2 \mathrm{~m}$ is considered. The plate is simple supported at all the four edges and the forcing function is acting at the centre of the plate transversely. Taking advantage of the symmetry of boundary conditions and loading one fourth of the plate is analysed. In each case the material properties are as follows:

\begin{tabular}{|l|l|}
\hline Material & Aluminium \\
\hline Modulus of Elasticity $(\mathrm{E})$ & $6.85 \times 10^{10} \mathrm{~N} / \mathrm{m}^{2}$ \\
\hline Shear Modulus $(\mathrm{G})$ & $2.64 \times 10^{10} \mathrm{~N} / \mathrm{m}^{2}$ \\
\hline Density $(\rho)$ & $2743.7 \mathrm{~kg} / \mathrm{m}^{3}$ \\
\hline Poisson's Ratio $(\mu)$ & 0.3 \\
\hline
\end{tabular}




\subsection{Study of Response at Different Distances from}

\section{Centre of Plate}

The plate with the above dimension and material property is analysed with the forcing function as mentioned earlier. The different mesh sizes used are from $6 \times 6(100 \mathrm{mmx} 100 \mathrm{~mm})$ to $48 \times 48(12.5 \mathrm{~mm} \times 12.5 \mathrm{~mm})$. The time integration is run for $200 \mu \mathrm{s}$ with the time step $0.082 \mu \mathrm{s}$. It is found that at $36 \times 36$ mesh $(16.6 \mathrm{~mm} \times 16.6 \mathrm{~mm})$ the results show acceptable convergence. The transverse displacement response (w) is studied at a distance $0.15 \mathrm{~m}, 0.3 \mathrm{~m}, 0.45 \mathrm{~m}$ and $0.6 \mathrm{~m}$ from the centre of the plate as is presented in Fig. 1 and Fig. 2. It is observed that the response becomes increasingly feeble as one moves away from the source; the pattern tallies with expected physical behaviour of the plate.

In addition, it is also observed that amplitude of displacement at a particular point keeps on increasing with time during the interval through which the force is active.
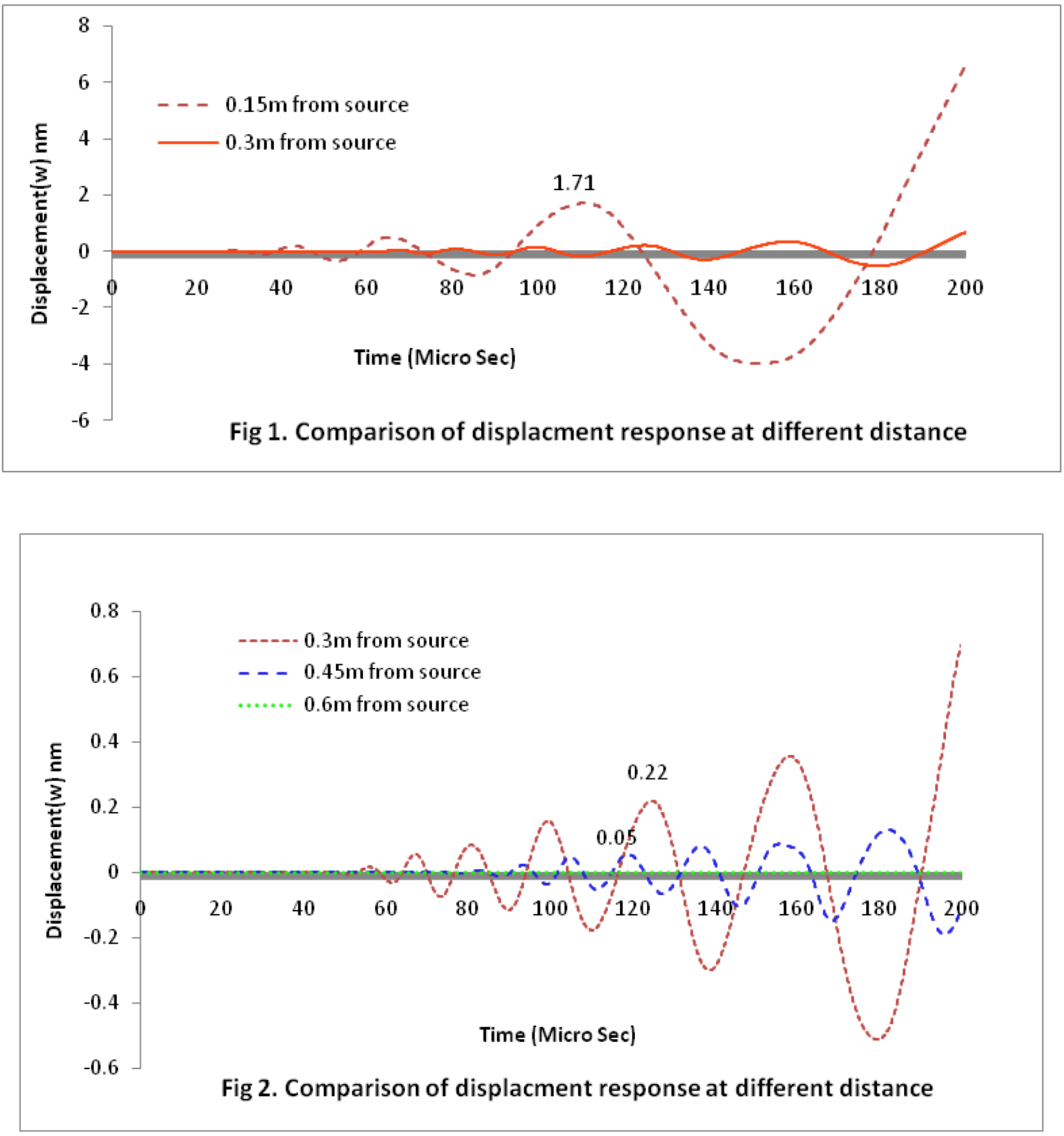


\subsection{Study of response at $0.25 \mathrm{~m}$ from centre of plate}

To validate the response pattern as obtained by the present formulation against the results available from wave propagation based approach [4], the same plate as in example 2.1 is studied for displacement (w) time history at $0.25 \mathrm{~m}$ from the centre of the plate. The response is presented in Fig. 3 and is compared with that at $0.254 \mathrm{~m}$ from the centre of a similar plate under an equivalent $\mathrm{AE}$ signal [4] as discussed earlier. The comparison shows that the displacement patterns are similar. Up to $80 \mu$ s it is found that the displacement amplitude does not reach any significant value. Beyond $80 \mu$ s the responses are observed with sharp peak amplitudes which are increasing with the progress of time. At about $140 \mu$ s sharp peak amplitude is observed and the peak value is about $0.51 \mathrm{~nm}$. This response amplitude is found to be almost equal to the amplitude as obtained in the study in Ref. [4].

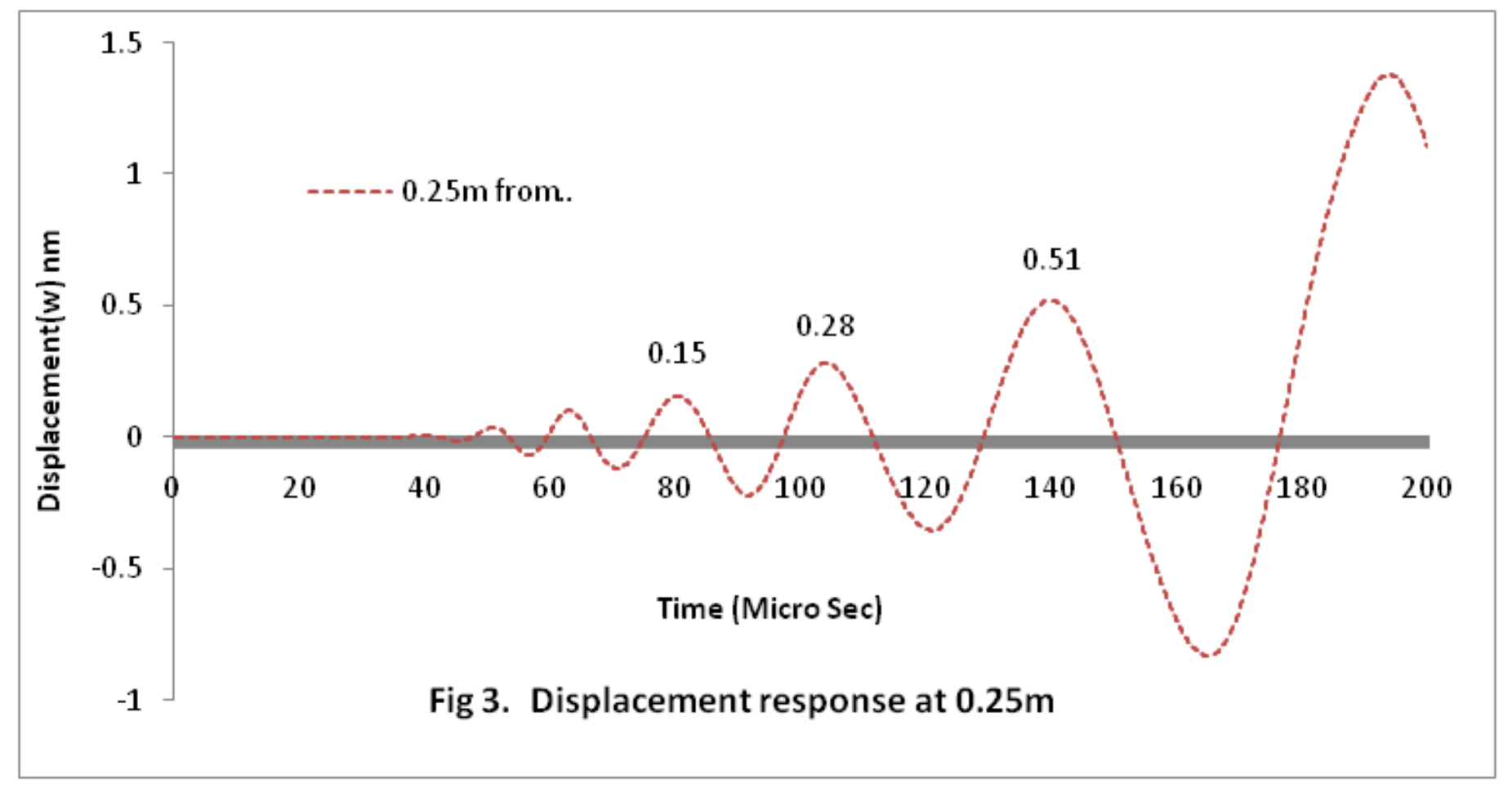

\section{CONCLUSIONS}

The response of an isotropic plate, treated as a vibration problem, shows considerable similarity with the response of the same plate treated as wave propagation problem. In the model numerical based FE technique is used. To check the efficacy of the model, convergence study is also done. Hence the numerical model developed for plate vibration for structural investigations can be considered as a fair alternative to wave propagation approach. Moreover, the following important conclusions can be made from the study:

The structural engineers are mostly interested in finding out damage through quantification where the proposed method of investigation can be useful.

The modal parameters may be extracted from the response data in the proposed vibration based approach, which may not be possible in wave propagation approach.

Standard response function which is used to represent crack can be modified according to the severity for further structural investigations.

The developed numerical model in FEM can also be generalized for structural investigations to study the health of the structure completely.

\section{REFERENCES}

[1] Ono, K., Ohtsu, M., "A generalized theory of acoustic emission and Green's functions in a half space", Journal of Acoustic Emission, V. 3, 1984, pp. 27-40

[2] Ohtsu, M., Ono, K., "The generalized theory and source representations of acoustic emission", Journal of Acoustic Emission, V. 5, 1986, pp. 124-133

[3] Gorman, M.R., Prosser, W.H., "AE source orientation by plate wave analysis", Journal of Acoustic Emission, Vol. 9, No. 4, 1990, pp. 283-286

[4] Gary, J., Hamstad, A., "On the far-field structure of waves generated by a pencil break on a thin plate", Journal of Acoustic Emission, V. 12, No. 3-4, 1994, pp. 157-170.

[5] Breckenridge, F., Proctor, T., Hsu, N., Fick, S., Eitzen, D., "Transient sources for acoustic emission work", Progress in Acoustic Emission V 1990, eds. K. Yamaguchi et al. JSNDI, Tokyo, 1990, pp. 20-37

[6] Hamstad, M.A., Gary, J., O'Gallagher, A., "A far-field acoustic emission waves by three-dimensional finite element modeling of pencil breaks on a thick plate", Journal of Acoustic Emission, 14, No. 2, 1996, pp. 103-114

[7] Hamstad, M.A., O'Gallagher, A., Gary, J., "Modeling of buried monopole and dipole sources of acoustic emission 
with a finite element technique", Journal of Acoustic Emission, V. 17, No. 3-4, 1999, pp. 97-110

[8] Prosser, W.H., Hamstad, M.A., Gary, J., O'Gallagher, A., "Reflections of $\mathrm{AE}$ waves in finite plates: finite element modeling and experimental measurements", Journal of Acoustic Emission, V. 17, 1999a, pp. 37-47

[9] Prosser, W.H., Hamstad, M.A., Gary, J., O'Gallagher, A., "Finite element and plate theory modeling of acoustic emission waveforms" Journal of Nondestructive Evaluation, V. 18, No. 3,1999b, pp. 83-90

[10] Sause, M.G.R., Horn, S., "Simulation of acoustic emission in planar carbon fiber reinforced plastic specimens", Journal of Nondestructive Evaluation, V. 29, No. 2, 2010a, pp. 123-142

[11] Sause, M.G.R., Horn, S., (2010b). "Simulation of lamb wave excitation for different elastic properties and acoustic emission source geometries", Journal of Acoustic Emission,V. 28, 2010b, pp. 109-121

[12] Sause, M.G.R., "Acoustic emission signal propagation in damaged composite structures", Journal of Acoustic Emission, V. 31, No. 1, 2013, pp. 1-18

[13] Sengupta, S., Datta, A.K.,Topdar, P., "Structural Damage Localisation by acoustic emission technique: A state of the art review", Latin American Journal of Solids and Structures, V. 12, 2015, 1565-1582

[14] Bathe, K.J., "Finite Element Procedures", Prentice Hall, 1996

[15] Topdar, P., Sheikh, A.H., Dhang, N., "Vibration characteristics of composites/sandwich laminates with piezoelectric layers using a refined hybrid plate model", International Journal of Mechanical Sciences, V. 49, 2001, pp. 1193-1203 